\title{
Infecção de trato urinário inferior por Citrobacter sp em cadela com hipoadrenocorticismo*
}

\section{Citrobacter sp lower urinary tract infection in a bitch with hypoadrenocorticism}

\author{
Marcy Lancia Pereira, ${ }^{* *}$ Maria Helena Souza Aguiar, ${ }^{* * *}$ Nicole Anzanello Meira Nercolini ${ }^{* * * *}$
}

\begin{abstract}
Resumo
Citrobacter sp. é um microrganismo frequentemente encontrado em vagina de cadelas, mas corresponde a menos de $3 \%$ do total de microrganismos isolados em urocultura. O hipoadrenocorticismo (HA) é uma doença endócrina incomum e que leva a poliúria e hipostenúria. O objetivo deste trabalho é relatar o caso de uma fêmea da espécie canina, da raça Teckel, 11 anos, atendida com queixa de urina de odor alterado há vários dias, além de vômito, diarreia e inapetência há três dias, e poliúria e polidipsia há 2 meses, com histórico de tratamento anterior com mitotano para hiperadrenocorticismo. O diagnóstico foi de ITU por Citrobacter sp., além de HA iatrogênico. Como destaques nos exames, relação sódio:potássio de 22,6 foi observada, sugestiva de HA, que foi confirmado por teste de estimulação com ACTH, além de urina inicialmente hipostenúrica (densidade 1,006), o que pode ser atribuído ao washout medular renal devido à hiponatremia causada pelo hipoadrenocorticismo. $\mathrm{O}$ tratamento foi feito com amoxicilina associada a ácido clavulânico por 20 dias, além da terapia específica para HA com prednisona e fludrocortisona, que foi continuado. A paciente recuperou-se bem, a densidade urinária aumentou após início do tratamento para HA e não houve recidiva da ITU em período de acompanhamento de 8 meses. Acredita-se que a baixa densidade urinária causada pelo HA tenha sido fator essencial para a ocorrência da ITU por Citrobacter.
\end{abstract}

Palavras-chave: cão, adrenal, cistite bacteriana, doença de Addison.

\begin{abstract}
Citrobactersp. is a normal constituent of bitches' vagina, but it is related to less than $3 \%$ of total isolated microorganism in uroculture. Hypoadrenocorticism (HA) is an endocrine disease uncommonly diagnosed that leads to polyuria and hypostenuria. The aim of this work is report the case of a Teckel bitch, 11 years old, attended with complaint of altered urinary smell for several days, besides vomiting, diarrhea and inappetence for three days, and polyuria and polydispsia for two months, with previous mitotane treatment for hyperadrenocorticism. The diagnosis was Citrobacter UTI, besides iatrogenic HA. The most important exam results include sodium:potassium ratio of 22.6, suggestive of HA, that was confirmed by ACTH stimulation test. Besides, hypostenuria (urinary specific gravity - USG - of 1.006) was noted, attributed to renal medular washout due to hyponatremia seen in HA. Therapy comprised amoxicillin associated with clavulanic acid for 20 days, in addition to specific therapy for AH with prednisone and fludrocortisone. The patient recovery well, USG increased after the beginning of the treatment for HA and there was no UTI reinfection for a 8 month period. It is believed that low USG due to HA was essential for the occurrence of Citrobacter UTI.
\end{abstract}

Keywords: dog, adrenal, bacterial cystitis, Addison disease.

\section{Introdução}

As infecções de trato urinário (ITU) acometem frequentemente os animais domésticos, sendo a cistite bacteriana a mais comum $(40 \%)$, seguida da incontinência urinária (24\%), urolitíase (18\%), dentre outras (Lulich et al., 2008). As ITUs ocorrem com maior incidência em fëmeas, devido à anatomia do seu sistema urogenital, que favorece a infecção por possuírem uma uretra curta e pela proximidade do ânus com as genitálias ou vias urinárias. As bactérias mais frequentemente isoladas são a Escherichia coli, Klebsiella pneumoniae, Pseudomonas aeruginosa e Enterobacter spp (Kogika et al., 1995; Ling et al., 2001; Senior, 2011; Carvalho, 2014; Barsanti, 2006).
Para diagnóstico definitivo e tratamento adequado, indica-se urocultura com antibiograma (Vasconcellos, 2012; Carvalho et al., 2014). Tais exames indicam a antibioticoterapia mais eficiente voltado ao agente patológico isolado na urina, tornando o tratamento seguro (Lulich e Osborne, 1995; Bartges, 2005; Barsanti, 2006; Kogika, 2009).

O hipoadrenocorticismo canino (síndrome de Addison) resulta da secreção hormonal adrenocortical (glicocorticoide e mineralocorticoide) insuficiente para manter as necessidades fisiológicas básicas do organismo (Boujon et al., 1994; ScottMoncrieff, 2007). Costuma ser uma endocrinopatia incomum e de difícil diagnóstico, já que os sinais clínicos mimetizam

${ }^{*}$ Recebido em 24 de abril de 2020 e aceito em 16 de julho de 2020.

**Docente, Universidade Federal de Santa Catarina (UFSC), Centro de Ciências Rurais, campus Curitibanos, Coordenadoria Especial de Biociências e Saúde Única, Brasil. E-mail: marcy.pereira@ufsc.br.

${ }^{* * *}$ Graduanda de Medicina Veterinária da Universidade Federal de Santa Catarina (UFSC), Centro de Ciências Rurais, campus Curitibanos, Brasil.

${ }^{* \star * *}$ Médica Veterinária autônoma, Blumenau, Santa Catarina, Brasil. 
doenças comuns na clínica de pequenos animais. Por este motivo, a doença normalmente é subdiagnosticada (Granados et al., 2011). Pode ser classificada em primária (mais comum), secundária e terciária (Romão e Antunes, 2012) e segundo Klein e Petenson (2010), os sinais clínicos aparecem somente quando mais de $85 \%$ das adrenais estão comprometidos. Na maioria dos casos, o motivo subjacente da destruição adrenal é idiopático ou imunomediado. Sobredosagem e/ou reações idiossincráticas em cães com hiperadrenocorticismo tratados com mitotano e trilostano também resultam em hipoadrenocorticismo temporário e permanente (Church, 2009). A incidência do hipoadrenocorticismo é relatada entre 0,36 a $0,5 \%$ de pacientes caninos, tendo maior prevalência em fêmeas jovens (Granados et al., 2011).

Este trabalho tem como objetivo relatar a ocorrência de infecção do trato urinário por Citrobacter sp. em uma fêmea idosa da espécie canina, com hipoadrenocorticismo iatrogênico.

\section{Relato do caso}

Foi encaminhada para o Serviço de Nefrologia e Urologia do Centro de Diagnóstico e Especialidades uma cadela Teckel, de 11 anos, $8,1 \mathrm{~kg}$, castrada, cujo proprietário se queixava de urina de odor alterado há vários dias, além vômito, diarreia e inapetência há três dias, e poliúria e polidipsia há dois meses. A médica veterinária que encaminhou a paciente informou suspeita de hipoadrenocorticismo iatrogênico devido a tratamento anterior feito com mitotano indicado por outro colega. À anamnese, tutor negou realização de cateterização transuretral prévia e apresentou ultrassonografia realizada na semana anterior, em que não havia alterações vesicais nem adrenais. Os rins direito e esquerdo mediram 4,2cm e $3,8 \mathrm{~cm}$, respectivamente, sem alterações em rim direito, e rim esquerdo com contorno discretamente irregular e bem definido, arquitetura do parênquima preservada, heterogeneidade de cortical e dilatação discreta de pelve.

Ao exame físico, a paciente apresentava desidratação leve (5\%), arritmia sinusal respiratória e sopro grau II em valva mitral. Foram prescritos inicialmente ranitidina $(2,2 \mathrm{mg} / \mathrm{kg}, \mathrm{BID}, \mathrm{SC})$, cloridrato de metoclopramida $(0,5 \mathrm{mg} / \mathrm{kg}, \mathrm{BID}, \mathrm{SC})$ e fluidoterapia com solução ringer lactato por via intravenosa e subcutânea (450 mL e $300 \mathrm{~mL}$, respectivamente), diariamente, até novas recomendações. Os exames laboratoriais solicitados, cuja coleta de material biológico ocorreu durante a consulta foram urinálise, urocultura e antibiograma, além de ureia, creatinina, sódio e potássio séricos (Tab. 1)

A colega encaminhante solicitara mensuração de sódio e potássio, com resultado de relação Na:K 22,6 (referência Na:K $\geq$ 27), o que permitiu à colega suspeitar de hipoadrenocorticismo. O teste de estimulação com ACTH sintético confirmou o diagnóstico e, assim, iniciou-se tratamento com prednisona (1 mg/kg BID) e fludrocortisona $(0,025 \mathrm{mg} / \mathrm{kg}$ BID) até novas recomendações.

Durante a primeira reavaliação, 10 dias após a consulta inicial, o tutor relatou melhora do estado geral e dos sinais gastrintestinais, exceto em relação ao odor da urina. O resultado de urocultura mostrou crescimento de Citrobacter sp. e, o antibiograma, demonstrou que o microorganismo era sensível aos seguintes antimicrobianos: Amoxicilina com ácido clavulânico, cefazolina, ceftiofur, cefepime, ceftriaxona, gentamicina e florfenicol. A bactéria cultivada mostrou resistência a ampicilina, ciprofloxacina, enrofloxacina, norfloxacina e penicilina G.

Suspendeu-se então a fluidoterapia e prescreveu-se amoxicilina associada a ácido clavulânico $(20 \mathrm{mg} / \mathrm{kg}$, BID por até 20 dias). Solicitou-se retorno após uma semana do início da antibioticoterapia para realização de coleta de urina para nova cultura e antibiograma, a fim de permitir tomada de decisão de continuação da terapia até o final dos 20 dias. Entretanto, a paciente retornou somente após 35 dias (Tabela 1). A cadela estava muito bem clinicamente, a antibioticoterapia havia sido realizada conforme prescrita e houve indicação apenas para continuação com o tratamento para hipoadrenocorticismo.

Na reavaliação 8 meses após diagnóstico inicial de infecção de trato urinário, a paciente apresentou-se bem, sem queixas. Os exames mostraram resultados normais para hemograma, bioquímica sérica e urinálise e, à urocultura, mais uma vez não houve crescimento bacteriano.

Tabela 1: Resultado dos exames complementares da paciente canina ao longo do acompanhamento médico veterinário, realizado no dia da consulta inicial, dez dias após e quarenta e cinco dias após

\begin{tabular}{|c|c|c|c|c|}
\hline Exame & Dia da consulta & $8^{\circ} \mathrm{dia}$ & $45^{\circ} \mathrm{dia}$ & Referência* \\
\hline \multirow{4}{*}{$\begin{array}{c}\text { Urinálise } \\
\text { (urina coletada por } \\
\text { cistocentese) }\end{array}$} & Densidade 1,006 & Densidade 1,036 & Densidade 1,034 & Densidade 1,015 a 1,045 \\
\hline & $\mathrm{pH} 6$ & $\mathrm{pH} 5,5$ & $\mathrm{pH} 7$ & $\mathrm{pH} 5,5$ a 7 \\
\hline & Nitrito +, proteína 1+ & Nitrito +, proteína 1+ & - & - \\
\hline & Bacteriúria 1+ & Leucócitos e bactérias raras & Leucócitos raros & - \\
\hline Cultura & Citrobacter sp & & Negativa & Negativa \\
\hline Creatinina & $1,91 \mathrm{mg} / \mathrm{dl}$ & & $1,07 \mathrm{mg} / \mathrm{dl}$ & 0,5 a $1,4 \mathrm{mg} / \mathrm{dl}$ \\
\hline Ureia & $62,86 \mathrm{mg} / \mathrm{dl}$ & & 72,4 mg/dl & 21,4 a $60,0 \mathrm{mg} / \mathrm{dl}$ \\
\hline Sódio & $131 \mathrm{mEq} / \mathrm{l}$ & $137 \mathrm{mEq} / \mathrm{l}$ & $143 \mathrm{mEq} / \mathrm{l}$ & 141,1 a $153 \mathrm{mEq} / \mathrm{L}$ \\
\hline Potássio & $5,8 \mathrm{mEq} / \mathrm{l}$ & $5,1 \mathrm{mEq} / \mathrm{l}$ & $4,9 \mathrm{mEq} / \mathrm{l}$ & 3,7 a $5,8 \mathrm{mEq} / \mathrm{L}$ \\
\hline Razão Na:K & 22,6 & 26,9 & 29,2 & ** \\
\hline PAS (Doppler) & $114,4 \mathrm{mmHg}$ & & & $<140 \mathrm{mmHg}$ \\
\hline
\end{tabular}

${ }^{*}$ Referência: laboratório SCAN, Campinas, SP.

**relação $\mathrm{Na}: \mathrm{K}<27$ sugere hipoadrenocorticismo. 


\section{Discussão e conclusões}

Clinicamente, as infecções bacterianas do trato urinário podem ou não apresentar sinais clínicos, sendo os mais comuns a disúria, polaquiúria, estrangúria, hematúria e incontinência urinária (Lulich e Osborne, 1995; Barsanti, 2006; Senior, 2011). Neste relato, na primeira consulta, a cadela não apresentou nenhum dos sinais clínicos supracitados, a única alteração notada relacionada à ITU foi odor da urina alterado. Ainda, mesmo a paciente tendo outros sinais inespecíficos como êmese, diarreia e inapetência, a suspeita do hipoadrenocorticismo ocorreu pelos dados fornecidos à anamnese de tratamento prévio para hiperadrenocorticismo com mitotano. Esta paciente não apresentava hipercalemia, seu potássio sérico apresentavase no limite superior, mas devido à hiponatremia, a relação sódio:potássio mostrou-se aumentada. De acordo com a literatura, a mensuração desses eletrólitos para cálculo da relação entre eles é teste de triagem para hipoadrenocorticismo e, quando menor que 23 , sugere que a confirmação da doença seja feita por meio da estimulação com ACTH sintético (Church, 2009; Granados et al., 2011; Lathan e Thompson, 2018), o que foi realizado para esta paciente.

Em relação à hipostenúria apresentada à primeira urinálise da cadela, sabe-se que pode ser justificada pela redução na reabsorção renal de água. Isso ocorre pela diminuição de hipertonicidade medular, também chamado de washout medular, devido à hiponatremia desencadeada pela insuficiência adrenocortical (Syme, 2014). Assim, tem-se urina diluída e poliúria, sinais compatíveis com hipoadrenocorticismo (Syme, 2014), predisponente ao aparecimento da ITU (Seguin et al., 2003; Weese et al., 2019).

A avaliação da creatinina sérica é importante quando se avaliam pacientes poliúricos. Normalmente, suspeita-se de doença renal crônica (DRC) quando há sinais clínicos compatíveis com poliúria e polidipsia por semanas a meses, associada a diminuição de densidade urinária e alteração ultrassonográfica (Syme, 2014). Esta paciente tinha sinais sugestivos de DRC, além de aumento de creatinina e ureia séricas observado ao primeiro exame. Entretanto, pela confirmação de hipoadrenocorticismo, conclui-se que a azotemia era de origem pré-renal, compatível com a desidratação leve constatada ao exame físico inicial pelos vômitos e poliúria apresentados. Corroborando esta análise, em exames posteriores, quando a paciente já estava em tratamento para a doença de base endócrina, observou-se que azotemia e densidade urinária baixa não mais foram notadas, devido à prevista recuperação da hipertonicidade medular renal e concentração urinária adequada.

\section{Referências}

BALL, K.R.; RUBIN, J.E.; CHIRINO-TREJO, M.; DOWLING, P.M. Antimicrobial resistance and prevalence of canine uropathogens at the Western-College of Veterinary Teaching Hospital, 20022007. Canadian Veterinary Journal, v.49, p. 985-990, 2008.

BARSANTI, J.A. Genitourinary infections. In: GREENE, C.E. Infectious diseases of the dog and cat. St Louis, Missouri: Saunders/Elsevier, 2006, p. 935-961.

BARTGES, J.W. Urinary Tract Infections. In: ETTINGER, S.J.; FELDMAN, E.C. (eds.), Textbook of veterinary internal medicine. Estados Unidos da América: Elsevier Saunders, 2005, p. 1800-1808.
A bactéria Citrobacter sp. é um bacilo gram-negativo entérico, oportunista, membro da família Enterobacteriaceae, comum ao trato intestinal de humanos e de outros animais (Tortora et al., 2012) e pode ser encontrada como parte da microbiota vaginal de cadelas (Barsanti, 2006; Grahan e Taylor, 2013).

Embora a Citrobacter possa estar associada a infecção de feridas cirúrgicas e de trato urinário (Tortora et al., 2012), a literatura traz que somente $3 \%$ das ITU em cães ocorrem devido a esse microrganismo (Kogika et al., 1995; Forrester et al., 1999; Norris et al., 2000; Ling et al., 2001; Ball et al., 2008; Carvalho et al., 2014). De acordo com Barsanti (2006) e Grahan e Taylor (2013), há necessidade de ocorrência de fatores predisponentes para que ocorra ascensão e colonização do TUI. Em humanos, há antiga associação desse microrganismo a doenças em adultos, especialmente idosos, debilitados e imunocomprometidos (Lipski et al., 1980) e também em crianças com más formações ou outras comorbidades (Barton e Walentik, 1982 e Gill e Schutze, 1999).

Não há, na medicina veterinária, descrição de fatores predisponentes ou desencadeantes específicos para a ocorrência da ITU por Citrobacter. Por outro lado, correlacionando a ITU aos mecanismos de defesa do hospedeiro, pode-se citar como fator importante a hiperosmolalidade urinária, pois essa faz parte das propriedades antimicrobianas da urina (Senior, 2011). Não se sabe, para esta paciente relatada, quais seriam os fatores relacionados à infecção por Citrobacter. O cateterismo, procedimento bastante corriqueiro na clínica e tido como fator predisponente (Senior, 2011), não foi realizado nesta cadela. Assim, talvez se possa considerar que a hipostenúria observada, relacionada ao hipoadrenocorticismo, tenha sido fator importante para a instalação da ITU.

De acordo com Weese et al. (2019), as infecções esporádicas ou não complicadas, como é o caso desta paciente, podem ou não ser tratadas com antibióticos. Empiricamente, a amoxicilina associada ao clavulanato é uma boa opção para tratamento quando não há possibilidade de aguardar resultado de cultura e antibiograma. Neste caso, devido à presença de sinais clínicos, optou-se pelo início da antibioticoterapia, que só ocorreu após resultado dos exames. A antibioticoterapia, por 3 a 5 dias, é indicada, embora haja pouco embasamento na literatura veterinária para tão curto período. Para a cadela relatada, a indicação da terapia foi por no máximo 20 dias, com necessidade de reavaliação de cultura e antibiograma após 7 dias de uso do antibiótico. Entretanto, como o tutor optou por fazer o tratamento até o final e não realizou coleta de urina para os exames solicitados, o período de antibioticoterapia acabou sendo extenso.

BARTON, L.L.; WALENTIK, C. Citrobacter diversus Urinary Tract Infection. American Journal of Diseases of Children, v. 136, n. 5, p. 467-468.

BOUJON, C.E.; BORNAND-JAUNIN, V.; SCHÄRER, V.; ROSSI, G.L.; BESTETTI, G.E. Pituitary gland changes in canine hypoadrenocorticism: a functional and immunocytochemical study. Journal of Comparative Pathology, v.111, n.3, p.287-95, 1994

CARVALHO, M.B. Semiologia do Sistema Urinário. In: FEITOSA, F.L.F. Semiologia Veterinária - A Arte do Diagnóstico. São Paulo: Roca, 2014, p. 427-448. 
CARVALHO, V.M.; SPINOLA, T.; TAVOLARI, F.; IRINO, K.; OLIVEIRA, R.M.; RAMOS, M.C.C. Infecções do trato urinário (ITU) de cães e gatos: etiologia e resistência aos antimicrobianos. Pesquisa Veterinária Brasileira, v.34, n.1, p.62-70, 2014.

CHURCH, D. B. Hipoadrenocorticismo canino. In: MOONEY, C.T.; PETERSON, M.E. Manual de endocrinologia canina e felina. São Paulo: Roca; 2009. p.207-216.

FORRESTER, S.D.; TROY, G.C.; DALTON, M.N.; HUFFMAN, J.W.; HOLTZMAN, G. Retrospective evaluations of urinary tract infection in 42 dogs with hyperadrenocorticism or diabetes mellitus or both. Journal of the Veterinary Internal Medicine, v. 13, p.577-560, 1999.

GILL, M.A.; SCHUTZE, G.E. Citrobacter urinary tract infections in children. The Pediatric Infectious Disease Journal, v.18, p. 889-892, 1999.

GRANADOS, J. L. MARTÍNEZ L. M. GALINDO, V. Hipoadrenocorticismo primario canino: reporte de caso. Revista de la Facultad de Medicina Veterinaria y de Zootecnia, v.1, n.58, p. 34-44, 2011.

KLEIN, S.C.; PETERSON, M.E. Canine hypoadrenocorticism: part I. The Canadian Veterinary Journal, v.51, p.63-9, 2010.

KOGIKA, M.M.; FORTUNATO, V.A.B.; MAMIZUKA, E.M.; HAGIWARA, M.K.; PAVAN, M.F.B.; GROSSO, S.N.A. Etiologic study of urinary tract infection in dogs. Brazilian Journal of Veterinary Research and Animal Science, v.32, n. 1, p.31-6, 1995.

KOGIKA, M.M. Recommendations for management of urinary tract infection in dogs. In: COMUNICAÇÃO APRESENTADA NO 34TH WORLD SMALL ANIMAL VETERINARY CONGRESS. 2009. Anais ... São Paulo: WSAVA, 2009, p.21-24.

LATHAN, P.; THOMPSON, A.L. Management of hypoadrenocorticism (Addison's disease) in dogs. Veterinary Medicine: Research And Reports, v.9, n.8, p. 1-10, 2018.

LING, G.V.; NORRIS, C.R.; FRANTI, C.E.; EISELE, P.H.; JOHNSON, D.L.; RUBY, A.L.; JANG, S.S. Interrelations of Organism Prevalence, Specimen Collection Method, and Host Age, Sex, and Breed among 8,354 Canine Urinary Tract Infections (1969-1995). Journal of Veterinary Internal Medicine, v.15, p.341-347, 2001.

LIPSKY, B.A.; HOOK, E.W.; SMITH, A.A, PLORDE, J.J. Citrobacter infections in humans: Experience at the Seattle Veterans Administration Center and a review of the literature. Reviews of Infectious Diseases, v. 2, p.746-760, 1980.
LULICH, J.P.; OSBORNE, C.A.; BARTGES, J.W.; LEKCHAROENSUK, C. Distúrbios do trato urinário inferior dos caninos. In: ETTINGER, S.J.; FELDMAN, E.C. Tratado de Medicina Interna Veterinária. São Paulo: Manole, 2008, p. 18411867.

LULICH, J.P.; OSBORNE, C.A. Bacterial infections of the urinary tract. In: ETTINGER, S.J.; FELDMAN, E.C. Textbook of Veterinary Internal Medicine. Philadelphia: Saunders, 1995, p.1775-1788.

NORRIS, C.R.; WILLIAMS, B.J.; LING, G.V.; FRANTI, C.E.; JOHNSON, D.L.; RUBY, A.L. Recurrent and persistent urinary tract infections in dogs: 383 cases (1969-1995). Journal of the American Animal Hospital Association, v. 36, p. 484-492, 2000.

ROMÃO, F.G.; ANTUNES, M.I.P.P. Hipoadrenocorticismo em cães: revisão. Revista Veterinária e Zootecnia, v.19, n.1, p.44-54, 2012.

SCOTT-MONCRIEFF, J.C. Canine hypoadrenocorticism: what's new? In: PROCEEDINGS OF 24TH NORTH AMERICAN VETERINARY CONFERENCE. 2007. Anais... Ithaca: NAVC; 2007.

SEGUIN, M.A.; VADEN, S.L.; ALTIER, C.; STONE, E.; LEVINE, J.F. Persistent urinary tract infections and reinfections in 100 dogs (1989-1999). Journal of Veterinary Internal Medicine, v.17, p. 622 631, 2003.

SENIOR, D. Urinary tract infection - bacterial. In: BARTGES, J.; POLZIN, D.J. Nephrology and Urology of Small Animals, Ames, lowa: Wiley-Blackwell, 2011, p.710-716.

SYME, H.M. Poliúria e polidipsia. In: ELLIOT, J.; GRAUER, G.F. Manual de Nefrologia e Urologia em Cães e Gatos. São Paulo: Roca, 2014, p. 9-28.

TORTORA, G.J.; FUNKE, B.R.; CASE, C.L. Classificação dos microrganismos. In: _. Microbiologia. Porto Alegre: Artmed, 2012, p. 273-298.

VASCONCELLOS, A.L. Diagnóstico de cistite em cães contribuição dos métodos de avaliação. 2012. 71 f. Dissertação (Mestrado) - Faculdade de Ciências Agrárias e Veterinárias - Universidade Estadual Paulista, campus de Jaboticabal, São Paulo, 2012.

WEESE, J.S.; BLONDEAU, J.; BOOTHE, D.; GUARDABASSI, L.G.; GUMLEY, N.; PAPICH, M.; JESSEN, L.R.; LAPPIN, M.; RANKIN, S.; WESTROPP, J.L.; SYKES, J. International Society for Companion Animal Infectious Diseases (ISCAID) guidelines for the diagnosis and management of bacterial urinary tract infections in dogs and cats. The Veterinary Journal, v.247, p.8-25, 2019. 